\title{
Prevalência de aglutininas anti-Leptospira interrogans em cães de Belo Horizonte, Minas Gerais, 2001 a 2002
}

[Prevalence of anti-Leptospira interrogans agglutinins in dogs from Belo Horizonte, Minas Gerais, Brazil, 2001 - 2002]

\author{
D.F. Magalhães ${ }^{1}$, J.A. Silva ${ }^{1}$; E.C. Moreira ${ }^{1}$, V.M.L. Wilke ${ }^{2}$, J.P.A. Haddad ${ }^{1}$, J.N.C. Meneses ${ }^{1}$ \\ ${ }^{1}$ Escola de Veterinária - UFMG \\ Caixa Postal 567 \\ 30123-970 - Belo Horizonte, MG \\ ${ }^{2}$ Centro de Controle de Zoonoses - Prefeitura de Belo Horizonte, MG
}

\begin{abstract}
RESUMO
Avaliou-se a prevalência de aglutininas anti-Leptospira interrogans nos cães recolhidos pelo Centro de Controle de Zoonoses nas nove regionais administrativas de Belo Horizonte, em diferentes épocas do ano. As áreas de prevalência de cães reagentes foram correlacionadas às áreas de saneamento ambiental, usando recursos georreferenciamento. As amostras de sangue dos 3417 cães, coletadas no período de setembro de 2001 a setembro de 2002, foram processadas pela técnica de soroaglutinação microscópica, encontrando-se $13,1 \%$ de positividade. As sorovariedades Canicola, Ballum, Pyrogenes e Icterohaemorrhagiae foram as mais prevalentes. As mais altas prevalências ocorreram nas regionais Centro-Sul $(22,5 \%)$, Leste $(19,1 \%)$ e Nordeste $(13,2 \%)$. A menor prevalência foi observada na Regional Pampulha (8,2\%). Os meses com maior percentual de cães reagentes foram dezembro/2001 e janeiro/2002, período de maior precipitação pluvial e temperaturas médias mais elevadas. As áreas de maior risco coincidiram com as regiões de vilas, favelas e bairros da periferia onde existia deficiência de saneamento ambiental.
\end{abstract}

Palavras-chave: cão, Leptospira interrogans, prevalência, distribuição espacial

\begin{abstract}
Prevalence of anti-Leptospira interrogans agglutinins in dogs captured by the Zoonosis Control Center, in nine administrative districts of Belo Horizonte city, throughout the year, was evaluated. The areas with positive prevalence were correlated to the areas of environmental sanitation, using sources of georeference. Blood samples from 3,417 dogs captured, were submitted to microscopic agglutination test from September 2001 to September 2002, showed 13.1\% of seropositivity. The most reactive serovars were Canicola, Ballum, Pyrogenes and Icterohaemorrhagiae. High prevalences were observed at CenterSouthern - 22.5\%, Eastern - 19.1\% and Northeastern - 13.2\% districts. Pampulha district got $8.2 \%$ of prevalence, the lowest percentage of positive reactions. High number of dog infection was observed during December, 2001 and January, 2002, characterized as rainy period and high environmental temperature. Villages, slums and suburbs with environmental sanitation deficiency are areas of high risk.
\end{abstract}

Keywords: dog, Leptospira interrogans, prevalence, spatial distribution

Recebido em 16 de março de 2005

Aceito em 1 de novembro de 2005.

E-mail: danifm1@yahoo.com.br 


\section{INTRODUÇÃO}

As leptospiroses estão amplamente distribuídas pelo mundo, podendo afetar várias espécies de animais domésticos, silvestres e o homem. Os roedores são portadores sadios de leptospiras; entre esses o rato de esgoto (Rattus norvegicus), o rato dos telhados (Rattus rattus) e o camundongo (Mus musculus) são considerados as principais fontes de infecção para o cão e o homem. $\mathrm{O}$ cão tem papel importante na transmissão da doença ao homem, por manter a leptospira por longo período em seus rins, podendo eliminá-la na urina sem apresentar sinais clínicos ou após obter melhora clínica. Esse fato se torna mais agravante devido aos hábitos domésticos desses animais e sua estreita relação com os humanos. No Brasil muitos autores detectaram aglutininas anti-leptospiras em soros de cães, entre eles Santa Rosa et al. (1970) em São Paulo, Caldas e Dória (1976) na Bahia e Favero et al. (2002) em diversas regiões brasileiras. Em Belo Horizonte, os inquéritos sorológicos caninos foram realizados por Reis (1972), Santa Rosa et al. (1974) e Rocha et al. (2000). As leptospiroses apresentam forte significado sócio-econômico-cultural e são exacerbadas por fatores como o crescimento desordenado de grandes centros urbanos, as migrações, as deficiências nas condições de saneamento básico e o acúmulo de lixo, que promovem a expansão de roedores. A persistência do agente na natureza e o elevado potencial de infecção são assegurados pela diversidade de identidades sorológicas, pela multiplicidade de espécies hospedeiras e pelo relativo grau de sobrevivência das leptospiras patogênicas no ambiente, sem parasitismo, ainda que essas não se multipliquem fora do organismo dos hospedeiros (Faine et al., 1999).

Informações sobre distribuição da leptospirose canina em Belo Horizonte fornecerão elementos sobre áreas diferenciadas de risco, que serão úteis para planejar ações de um programa eficaz para controle dessa zoonose, permitindo intervenções saneadoras amplas nos locais de maior risco. Nessa cidade é comum encontrar nas ruas animais vadios, principalmente cães. Muitos são semidomiciliados, outros são totalmente abandonados e podem servir de fonte de infecção e dispersão da doença.
O presente estudo teve como objetivo determinar a prevalência de aglutininas anti-Leptospira interrogans em cães de Belo Horizonte em diferentes épocas do ano, correlacionando as áreas de prevalência de cães reagentes com áreas de saneamento ambiental.

\section{MATERIAL E MÉTODOS}

O trabalho foi desenvolvido nas nove regionais administrativas da Prefeitura Municipal de Belo Horizonte (PBH), de setembro de 2001 a setembro de 2002.

Para estimar a prevalência de cães sororreagentes às sorovariedades de Leptospira interrogans, utilizou-se o valor mediano da proporção $(\mathrm{p}=0,032)$, baseada no trabalho de Santa Rosa et al. (1974), que verificaram em Belo Horizonte $5,9 \%$ de cães reagentes, sendo $3,2 \%$ positivos para Icterohaemorragiae. Como essa sorovariedade foi a mais freqüente e representa grande importância em saúde pública, foi utilizada no cálculo como prevalência préestimada no município. Aplicou-se a fórmula para estudo de prevalência do Centro Panamericano de Zoonoses (Procedimientos... 1979), com $95,0 \%$ de grau de confiança e erro de 18,0\%. Dessa forma obteve-se uma amostra de 3191 cães, que foi fixada em 3500 animais para evitar que possíveis perdas de materiais pudessem interferir no resultado final. As amostras foram distribuídas proporcionalmente por regional, e os cães recolhidos pelo Centro de Controle de Zoonoses de Belo Horizonte (CCZ$\mathrm{BH}$ ), onde foram classificados, de acordo com a forma de apreensão, em cães de captura (sem endereço $n=2625$ ) e de busca domiciliar (com endereço completo $n=875$ ), ambos sem histórico de vacinações contra a leptospirose.

As amostras foram processadas pela técnica de soroaglutinação microscópica (SAM) (Ryu, 1970). Utilizaram-se, como antígenos, culturas vivas de leptospiras Canicola, Icterohaemorrhagiae, Pomona, Ballum, Pyrogenes, Autumnalis, Australis e Tarassovi, com cinco a sete dias de cultivo em meio líquido de Ellinghausen. A SAM foi considerada positiva quando ocorreu, no mínimo, 50,0\% de aglutinação, em diluições iguais ou superiores a $1: 200$. 
Verificou-se a associação dos cães positivos com os fatores de risco como saneamento e controle de roedores comparativamente através dos dados das regionais da $\mathrm{PBH}$. Essa comparação foi feita de forma descritiva, verificando a distribuição espacial dos casos positivos e das aglutininas anti-Leptospira interrogans mais prevalentes em cada regional e o mapeamento das regiões com ausência de rede de esgoto e coleta de lixo.

Para organização do banco de dados, foi utilizado o programa Epi-info versão 6.02 (Dean et al., 1995). Para a distribuição espacial dos cães positivos e das aglutininas anti-leptospiras, foram processados mapas utilizando-se os softwares MapInfo...(2004). Os bancos de dados para confecção dos mapas foram cedidos pela Prodabel e pelo Grupo Gerencial de Saneamento (GGSAN) da PBH. As diferenças de freqüências entre o tipo de apreensão dos cães foram analisadas pelo teste do $\chi^{2}$ (Sampaio 1998), fixando-se o nível de significância em $(\mathrm{P}<0,05)$ e processando as estatísticas na planilha eletrônica Excel versão 97. Para obtenção das Odds Ratio dos diferentes fatores de risco, foram utilizados modelos de regressão logística (Dohoo et al., 2003).

\section{RESULTADOS E DISCUSSÃO}

Foram processadas 3417 amostras de soros de cães, sendo 2589 de cães de busca domiciliar e 828 de cães de captura. Destas, $448(13,1 \%)$, apresentaram-se positivas na SAM para uma ou mais sorovariedades de Leptospira interrogans, com títulos que variaram de 1:200 a 1:25.600 (Tab. 1). Os maiores títulos foram para Canicola, Ballum, Pyrogenes e Icterohaemorrhagiae.

Tabela 1. Distribuição dos cães positivos para Leptospira interrogans de acordo com a forma de apreensão nas diferentes regionais da Prefeitura de Belo Horizonte, de setembro de 2001 a setembro de 2002

\begin{tabular}{|c|c|c|c|c|c|c|c|c|}
\hline \multirow{2}{*}{ Regional } & \multicolumn{2}{|c|}{ Amostra examinada } & \multicolumn{6}{|c|}{ Número de cães positivos à SAM } \\
\hline & BD & CAP & BD & $\%$ & CAP & $\%$ & Total & $\%$ \\
\hline Centro-Sul & 131 & 38 & 20 & 52,6 & 18 & 47,4 & 38 & 22,5 \\
\hline Leste & 295 & 92 & 54 & 73,0 & 20 & 27,0 & 74 & 19,1 \\
\hline Nordeste & 321 & 103 & 32 & 57,1 & 24 & 42,9 & 56 & 13,2 \\
\hline Norte & 362 & 120 & 36 & 60,0 & 24 & 40,0 & 60 & 12,5 \\
\hline Oeste & 224 & 74 & 24 & 64,9 & 13 & 35,1 & 37 & 12,2 \\
\hline Venda Nova & 345 & 115 & 25 & 43,9 & 32 & 56,1 & 57 & 12,4 \\
\hline Barreiro & 348 & 111 & 35 & 67,3 & 17 & 32,7 & 52 & 11,3 \\
\hline Noroeste & 352 & 110 & 30 & 57,7 & 22 & 42,3 & 52 & 11,1 \\
\hline Pampulha & 204 & 65 & 12 & 54,5 & 10 & 45,5 & 22 & 8,2 \\
\hline Total & 2589 & 828 & 268 & 59,9 & 180 & 40,1 & 448 & 13,1 \\
\hline
\end{tabular}

$\mathrm{SAM}=$ soroaglutinação microscópica; $\mathrm{BD}=$ cães apreendidos por busca domiciliar; $\mathrm{CAP}=$ cães apreendidos por captura .

De acordo com a forma de apreensão, verificouse maior prevalência entre os cães de captura $(\mathrm{P}<0,001)$. Possivelmente essa maior prevalência deve-se ao fato de os animais de rua estarem mais expostos às fontes de contaminação, além de, provavelmente, não serem vacinados contra a leptospirose.

A Fig. 1 apresenta a distribuição espacial dos cães amostrados. Os cães de busca domiciliar positivos à SAM, para uma ou mais sorovariedades de Leptospira interrogans, foram georreferenciados nas nove regionais do município de Belo Horizonte por desenhos coloridos, na posição exata do logradouro. Os cães capturados, por não terem endereço completo, foram georreferenciados pela prevalência encontrada em cada regional por sombreamento. A localização geográfica dos cães negativos à SAM foi representada por pontos negros; observa-se ampla distribuição desses animais em todas as regionais. 

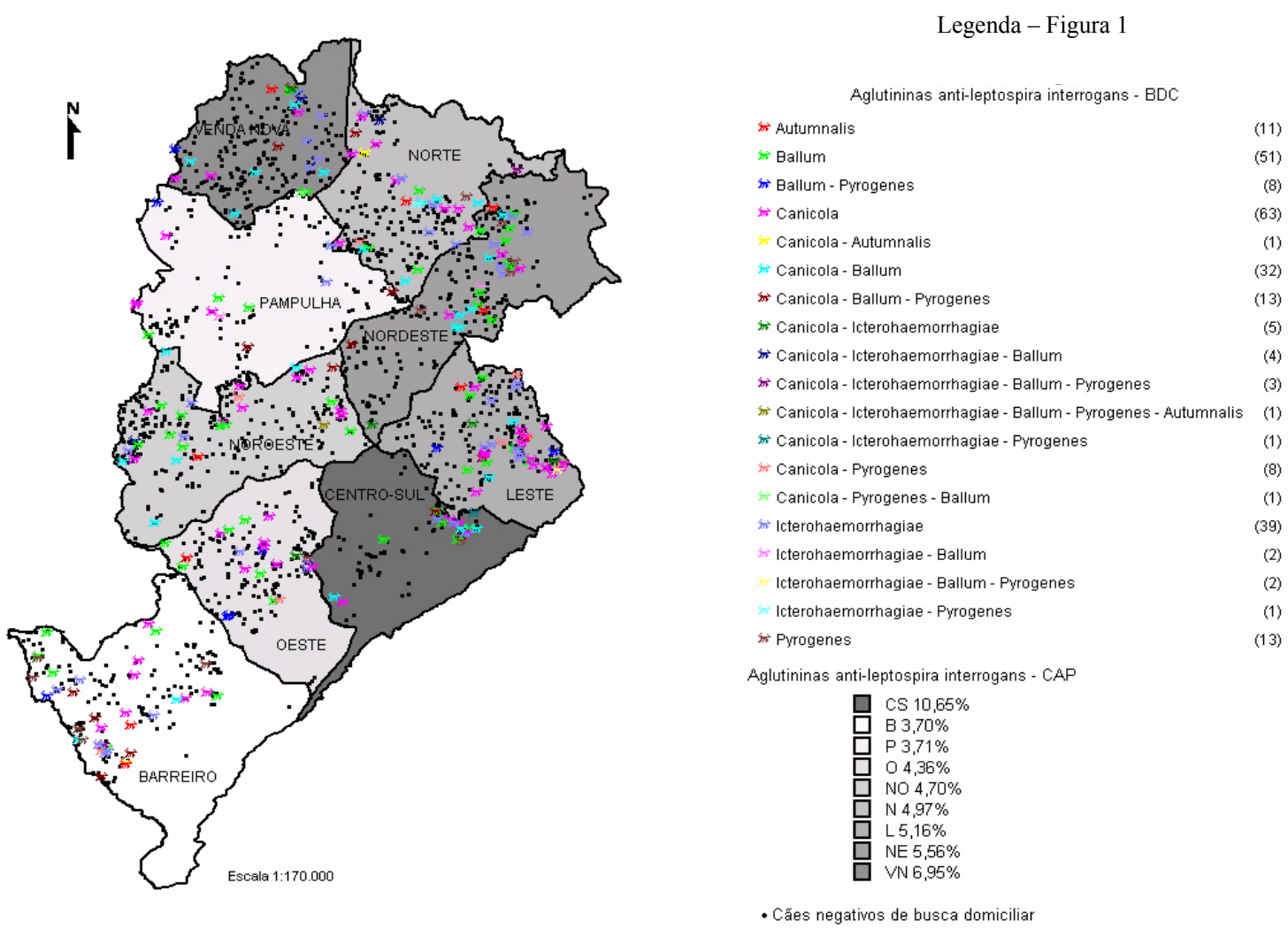

Figura 1. Distribuição dos cães nas regionais de Belo Horizonte - MG, setembro de 2001 a setembro de 2002.

A Regional Centro-Sul tem o menor número de cães em relação às outras áreas de Belo Horizonte e é considerada, segundo o índice de vulnerabilidade à saúde calculado pela Secretaria Municipal de Saúde, (a regional onde $75,0 \%$ da população humana se encontra na categoria de baixo risco (Tab. 2). Apesar disso, essa foi a regional que proporcionalmente apresentou $\mathrm{O}$ maior número de soros de cães positivos à SAM. Esse resultado, provavelmente, ocorreu porque os casos positivos se concentraram nas áreas de vilas e favelas existentes nessa regional. $\mathrm{O}$ aglomerado da Serra, do qual faz parte a favela do Cafezal, deteve o maior percentual de cães reagentes da Regional Centro-Sul. A segunda regional em percentual dos positivos foi a Leste, com as mesmas características de distribuição encontradas na Centro-Sul, predominância dos reagentes nas periferias e favelas da região, com destaque para os bairros Taquaril, Alto Vera Cruz e Novo Horizonte. Como essa regional possui mais da metade da população $(55,1 \%)$ classificada nas categorias de médio e elevado risco (Tab. 2), houve maior dispersão dos casos em outros bairros da regional, o que não foi observado na Regional Centro-Sul. A Pampulha foi a regional onde se observou a menor prevalência de aglutininas anti-Leptospira interrogans, com uma distribuição de reagentes mais uniforme em relação aos bairros, o que pode estar relacionado ao fato de essa regional ter $77,8 \%$ de seus moradores enquadrados nas categorias de baixo e médio risco à saúde. Nessa área, as favelas não representam um fator relevante em termos de expansão física, pois se apresentam de forma dispersa. O menor número de reações à Leptospira interrogans nessa regional demonstra o papel que esse tipo de moradia representa em termos de distribuição espacial da doença, conforme verificado nos estudos de Figueiredo et al. (2001). A Pampulha foi a única regional que não apresentou casos humanos de leptospirose. $\mathrm{O}$ conjunto habitacional Confisco deteve a maior 
porcentagem de cães reagentes da regional Pampulha. Na regional Nordeste, os maiores percentuais de soros positivos foram observados no Conjunto Paulo VI, na vila Belmonte e nos bairros Ribeiro de Abreu e São Marcos. A Regional Norte apresentou maior prevalência no Conjunto Jardim Felicidade e na vila São Tomáz. $\mathrm{Na}$ Regional Oeste, os cães reagentes se concentraram nos bairros Estrela Dalva, Madre Gertrudes e Jardim América. Na Regional Venda Nova, ocorreu uma dispersão mais acentuada de reagentes, com maior número nos bairros Jardim dos Comerciários e Céu Azul. A Regional Barreiro, por possuir uma grande área não habitada, devido às reservas de matas da Mannesman, apresentou uma distribuição de reagentes em sua periferia, principalmente no Vale do Jatobá, Mangueiras e Independência. A Regional Noroeste concentrou os reagentes nas Vilas Sumaré e São José e nos bairros Califórnia e Jardim Alvorada.

Tabela 2. População por categoria de risco $^{1}$ segundo regionais da Prefeitura de Belo Horizonte, 2000

\begin{tabular}{|c|c|c|c|c|c|c|c|c|}
\hline \multirow{3}{*}{ Regional } & \multicolumn{8}{|c|}{ Categoria de Risco ${ }^{1}$} \\
\hline & \multicolumn{2}{|c|}{ Muito elevado } & \multicolumn{2}{|c|}{ Elevado } & \multicolumn{2}{|c|}{ Médio } & \multicolumn{2}{|c|}{ Baixo } \\
\hline & Número & $\%$ & Número & $\%$ & Número & $\%$ & Número & $\%$ \\
\hline Barreiro & 18.593 & 7,1 & 102.851 & 39,2 & 134.933 & 51,5 & 5.817 & 2,2 \\
\hline Centro Sul & 31.473 & 11,8 & 26.756 & 10,1 & 6.943 & 2,6 & 200.831 & 75,5 \\
\hline Leste & 18.121 & 7,3 & 47.658 & 19,1 & 89.510 & 35,9 & 93.805 & 37,7 \\
\hline Nordeste & 15.065 & 5,5 & 79.677 & 29,1 & 125.968 & 46,0 & 53.350 & 19,5 \\
\hline Noroeste & 17.567 & 5,2 & 54.096 & 16,0 & 169.249 & 50,2 & 96.439 & 28,6 \\
\hline Norte & 16.829 & 8,7 & 85.834 & 44,3 & 83.863 & 43,3 & 7.238 & 3,7 \\
\hline Oeste & 16.654 & 6,2 & 81.451 & 30,3 & 76.481 & 28,5 & 94.112 & 35,0 \\
\hline Pampulha & 8.037 & 5,6 & 23.513 & 16,5 & 42.957 & 30,1 & 68.095 & 47,8 \\
\hline Venda Nova & 15.558 & 6,4 & 101.764 & 41,6 & 119.707 & 48,9 & 7.537 & 3,1 \\
\hline Total & 157.897 & 7,1 & 603.600 & 27,0 & 849.611 & 38,0 & 627.224 & 28,0 \\
\hline
\end{tabular}

${ }^{1}$ Fonte: Gerência de Epidemiologia e Informação - SMSA/Censo Populacional IBGE - 2000. Comunicação pessoal. Segundo índice de vulnerabilidade à saúde.

Assim, verifica-se que os principais focos de infecção por Leptospira interrogans se concentraram nas áreas de periferia das regionais, principalmente nas favelas. Essas áreas são caracterizadas por não contarem com medidas de saneamento ambiental, como rede de esgoto, coleta de lixo e controle de roedores, expondo a população residente e os animais que vivem nesses locais ao risco de contraírem diversas doenças infecciosas e parasitárias, especialmente aquelas veiculadas pela água contaminada e pelo lixo, o que foi observado por Breilh e Granda, (1986) em Quito, Equador. A distribuição da infecção por Leptospira interrogans em áreas de favelas e bolsões de pobreza, foi relatada por Alves et al. (2000) em cães no município de Patos, na Paraíba e por Figueiredo et al. (2001), em Belo Horizonte, em casos de leptospirose humana.

A Fig. 2 apresenta a distribuição espacial das áreas não atendidas por rede de esgoto, com sobreposição dos cães positivos para Leptospira interrogans. Nota-se que a maior parte dos cães positivos era proveniente de locais com deficiência de rede de esgoto, principalmente nas áreas de vilas e favelas. A variável coleta de lixo não mostrou correlação com a infecção por Leptospira interrogans (Fig.3). A não sobreposição dos cães reagentes nas áreas de deficiência de coleta de lixo pode ser explicada pelo fato de a $\mathrm{PBH}$ atender com serviços de limpeza aproximadamente $95,0 \%$ da população belo-horizontina. $\mathrm{O}$ percentual médio da população atendida por coleta de lixo em vilas e favelas é de $70,0 \%$, sendo a coleta de resíduos domiciliares nesses locais realizada por caminhões e garis que se deslocam porta a porta, com carrinhos de mão (Plano..., 2004). O controle do lixo urbano é de extrema importância para se evitar o surgimento de novos focos de infecção por Leptospira interrogans. $\mathrm{O}$ fato de essa variável não ter sido expressiva neste trabalho não diminui a responsabilidade da população e dos serviços de saúde em investir em projetos de limpeza, higiene, principalmente em terrenos baldios $\mathrm{e}$ em áreas intra $\mathrm{e}$ peridomiciliares e em projetos de educação sanitária. 


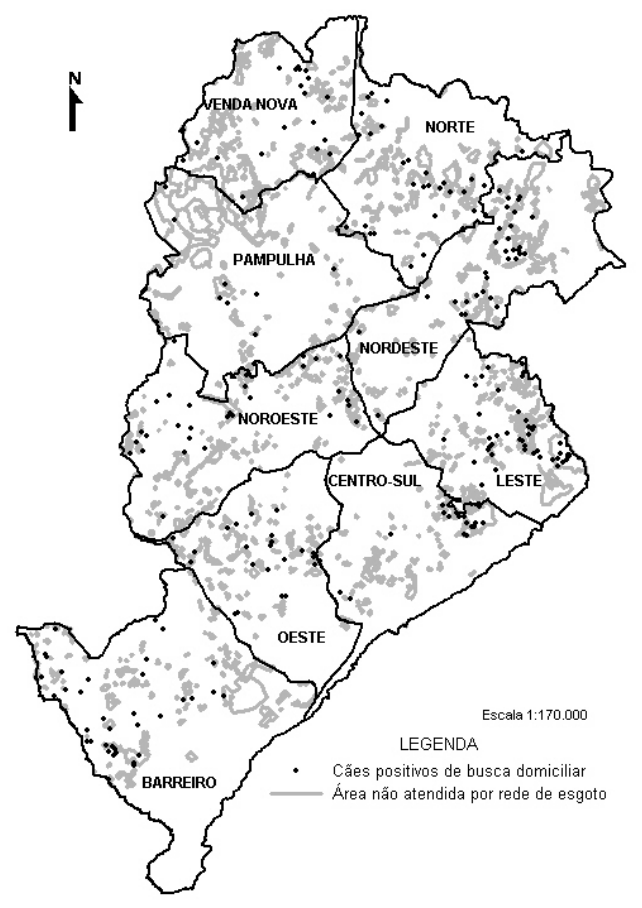

Figura 2. Distribuição dos cães positivos para $L$. interrogans em relação às áreas não atendidas por rede de esgoto em Belo Horizonte, MG, 2001 - 2002.

Quando se utiliza a regressão logística para análise das regionais, nota-se que, ao se considerar a Regional Barreiro como referência, pode-se, pela Odds Ratio, verificar que o fato de o cão ser domiciliado ou ser capturado na Regional Centro-Sul aumenta em 2,25 vezes a chance de esse animal ter infecção por Leptospira interrogans. Da mesma forma, o fato de o cão ser domiciliado ou ser capturado na Regional Pampulha representa um risco de 0,69 , fazendo com que o local de moradia possa ser apontado como um fator protetor. Isso pode ser explicado mais uma vez pelas características sócio-econômicas e de saneamento ambiental de cada regional.

As sorovariedades de Leptospira interrogans mais prevalente foram: Canicola $(7,0 \%)$ e Ballum $(6,1 \%)$, seguidas por Pyrogenes $(3,2 \%)$, Icterohaemorrhagiae $(2,9 \%)$, Autumnalis $(0,6 \%)$, Pomona $(0,3 \%)$, Australis $(0,3 \%)$ e Tarassovi $(0,1 \%)$. Como hipótese deste trabalho, já era esperada maior prevalência para Canicola, pois essa sorovariedade, segundo Hagiwara et al.

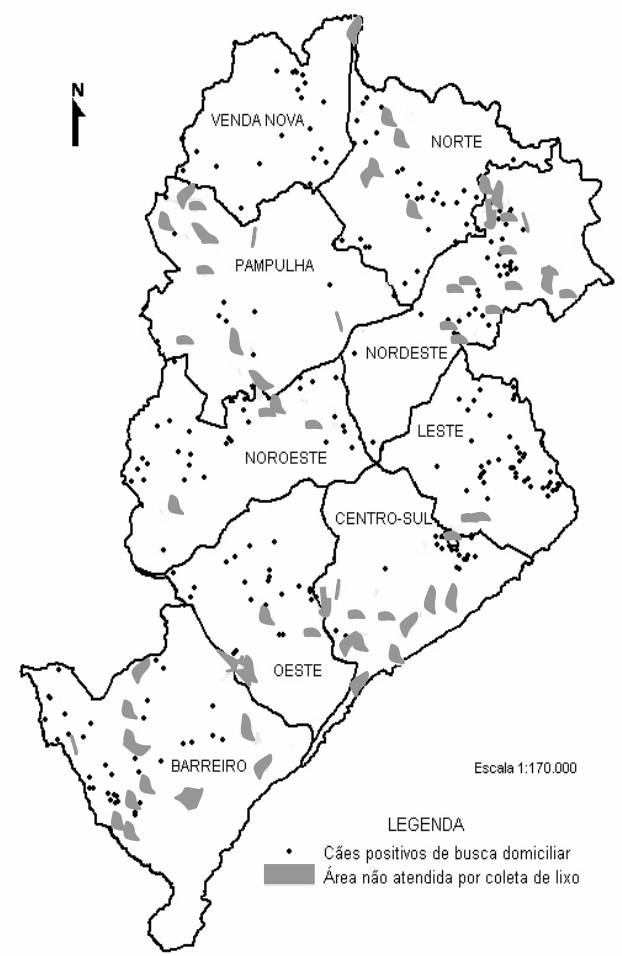

Figura 3. Distribuição dos cães positivos para $L$. interrogans em relação às áreas não atendidas por coleta de lixo em Belo Horizonte, MG, 2001 - 2002.

(2004), tem no cão seu hospedeiro natural e tem sido descrita como a mais prevalente nessa espécie (Caldas e Dória, 1976; Ávila et al., 1980; Ávila et al., 1998; Modolo et al., 1999). A importância de se encontrar altos títulos de anticorpos e alta prevalência para a sorovariedade Canicola relaciona-se ao quadro de leptospirúria que pode persistir por vários meses (Alves et al 2000). Em infecções pela sorovariedade Canicola, o estado de portador real ocorre em maiores proporções quando comparado com a infecção pela sorovariedade Icterohaemorrhagiae.

A sorovariedade Ballum foi a segunda mais prevalente em Belo Horizonte, o que também foi observado por Ávila et al. (1980) em Jaboticabal. Em Buenos Aires, Rosseti et al. (1999) e Rocha et al. (2000), em Belo Horizonte, encontraram o sorogrupo Ballum como o mais freqüente. A alta prevalência das aglutininas anti-Ballum indicaria contato recente desses cães com roedores infectados, principalmente com camundongos (Mus musculus), demonstrando possível 
contaminação intradomiciliar Por isso, as ações de um programa de controle para leptospirose devem envolver também limpeza e desratização intradomiciliar, além das demais medidas preconizadas no Manual... (1995). Atenção especial deve ser dada à educação em saúde, ato de grande importância quando se trata de leptospirose. As condições precárias de moradia e a grande exposição aos fatores de risco em que vive grande parte das pessoas refletem um nível baixo de educação dessa população, associado à ineficiência de infra-estrutura.

De acordo com os dados do Setor de Epidemiologia da Secretaria Municipal de Saúde de Belo Horizonte, no período de setembro de 2001 a setembro de 2002, foram recebidas 70.775 solicitações para controle de roedores nas nove regionais, sendo que a Nordeste foi responsável por $44,8 \%$ das solicitações, seguida pela Noroeste $(15,8 \%)$ e Oeste $(10,9 \%)$. A Regional Venda Nova foi a de menor índice de solicitações, sendo responsável por apenas 1,5\% das chamadas. A Regional Nordeste não foi a que apresentou maior prevalência de cães positivos, mas, das quatro sorovariedades georreferenciados em Belo Horizonte, Ballum foi a que teve maior distribuição espacial nessa regional, provavelmente, devido à presença de roedores, o que pode ser justificado pelo maior número de solicitações da comunidade feitas ao
Serviço de Controle de Zoonoses. A associação de casos de leptospiroses com a presença de roedores sinantrópicos também foi verificada por Barcellos et al. (2003) no Rio Grande do Sul.

Em infecção por leptospiras, a estação do ano é uma variável importante que deve ser levada em consideração, pois temperaturas amenas, em torno de $28^{\circ} \mathrm{C}$, e umidade relativa do ar elevada, favorecem a sobrevivência de leptospiras. Em épocas de alta precipitação pluvial, esses fatores, associados à ineficácia dos sistemas de drenagens da água, facilitariam o contato do cão com o agente. Os meses com maior prevalência de cães reagentes à Leptospira interrogans e os índices de precipitação pluvial são mostrados na Fig. 4. Nota-se que os maiores índices de infecção concentraram-se nos meses de chuva: dezembro/2001 (21,9\%), janeiro/2002 (21,7\%) e setembro/2001 (20,4\%). Ávila et al. (1998), em Pelotas, encontraram maior índice de infecção nos meses de março, agosto, setembro, outubro e novembro, correspondendo aos períodos de maiores índices pluviométricos naquele município.

Os resultados deste trabalho apontam a necessidade de estratégias diferenciadas de acordo com as áreas de risco de infecção por Leptospira interrogans no município de Belo Horizonte.

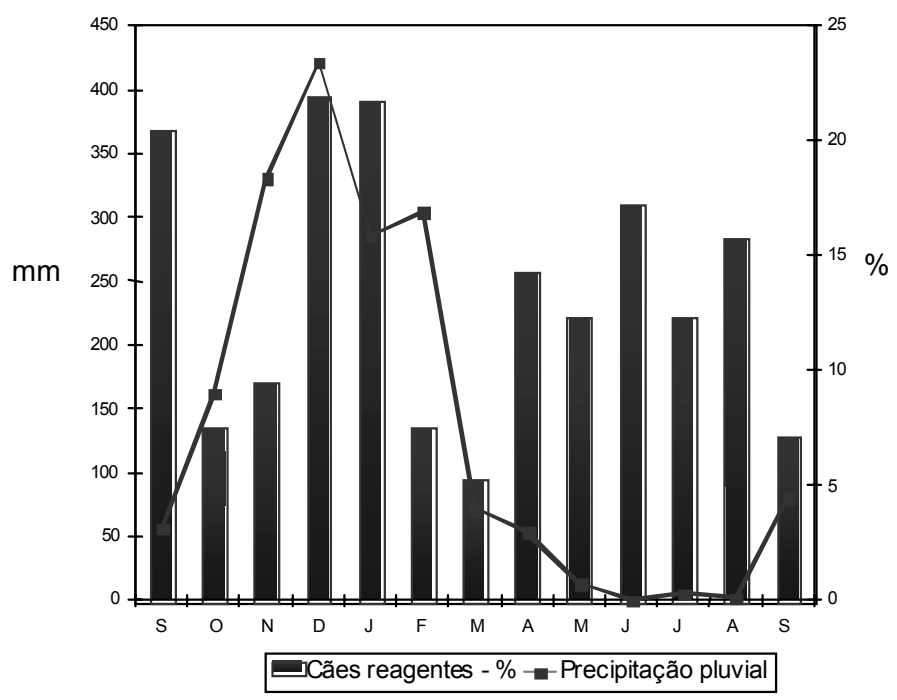

Figura 4 Distribuição dos cães positivos para Leptospira interrogans de acordo com o mês do ano e os índices de precipitação pluvial média em Belo Horizonte ${ }^{1}$, setembro de 2001 a setembro de 2002.

${ }^{1}$ Fonte: $5^{\circ}$ Distrito de Meteorologia do Ministério da Agricultura, Pecuária e Abastecimento - Comunicação pessoal. 


\section{REFERÊNCIAS BIBLIOGRÁFICAS}

ALVES, C.J.; ANDRADE, J.S.L.; VASCONCELLOS, S.A. et al. Avaliação dos níveis de aglutininas anti-leptospira em cães no município de Patos - PB, Brasil. Rev. Bras. Ciênc. Vet., v.7, p.1721,2000

ÁVILA, F.A.; PINTO, G.C.; PECEGO, C.E.V. et al. Freqüência de aglutininas anti-leptospira em cães do município de Jaboticabal, Brasil. In: ENCONTRO DE PESQUISAS VETERINÁRIAS, 5., 1980, Jaboticabal, Resumos... Jaboticabal, 1980. p.68.

ÁVILA, M.O.; FURTADO, L.R.I.; TEIXEIRA, M.M. et al. Aglutininas anti-leptospíricas em cães na área de influência do Centro de Controle de Zoonoses, Pelotas, RS, Brasil, no ano de 1995. Ciênc. Rural, Santa Maria), v.28. p.107-110, 1998.

BARCELLOS, C.; LAMMERHIRT, C.B.; ALMEIDA, M.A.B. et al. Distribuição espacial da leptospirose no Rio Grande do Sul, Brasil: recuperando a ecologia dos estudos ecológicos. $\mathrm{Cad}$. Saude Publica. v 19, p.1283-1292, 2003.

BREILH, J.; GRANDA, E. Investigação da saúde na sociedade: guia pedagógico sobre um novo enfoque do método epidemiológico. São Paulo: Instituto de Saúde/ ABRASCO, 1986. 215p.

CALDAS, E.M.; DÓRIA, J.D. Inquérito sorológico para leptospirose em Canis familiares, na cidade de Salvador. In: CONGRESSO BRASILEIRO DE MEDICINA VETERINÁRIA, 15, 1976, Rio de Janeiro. Anais... Rio de Janeiro: [s.n.] 1976. p.99.

DEAN, A.G.; DEAN J.A.; BURTON A.J. et al. EPIINFO, a word processing, date base and statistis program for epidemiology on microcomputers: version 6.02 Georgia: USD, 1995

DOHOO, I.; MARTIN, W.; STRYHN, H. Veterinary epidemiologic research. Charlottetown: AVC, 2003. $706 \mathrm{p}$.

FAINE, S.; ADLER, B.; BOLIN, C.; PEROLAT, P. Leptospira and leptospirosis. 2.ed. Austrália: MediSci, 1999. 272p.

FAVERO, A.C.M.; PINHEIRO, S.R.; VASCONCELLOS, S.A. et al. Sorovares de leptospiras predominantes em exames sorológicos de bubalinos, ovinos, caprinos, eqüinos suínos e cães de diversos estados brasileiros. Ciênc. Rural. (Santa Maria), v.32, p.613-619, 2002.

FIQUEIREDO, C.M.; MOURÃO, A.C.; OLIVEIRA, M.A.A. et al. Leptospirose humana no município de Belo Horizonte, Minas Gerais, Brasil: uma abordagem geográfica. Rev. Soc. Bras. Med. Trop., v.34, p.331338, 2001.
HAGIWARA, M.K; LUSTOSA, M.; KOGIKA, M.M. Leptospirose canina. Vetnews, v.4, p.1-3, 2004.

MANUAL de raiva e leptospirose. Informe técnico. Belo Horizonte: Secretaria de Estado de Saúde de MG, 1995. 78p.

MAPINFO professional Versão 6.0. Disponível em: $<\mathrm{http} / / /$ www.mapinfo.com. html $>$. Acesso em: $12 \mathrm{de}$ dezembro de 2004.

MODOLO, J.R., LANGONI, H.; SHIMABUKURU, F.H. et al. Inquérito soroepidemiológico para leptospirose canina no município de Botucatu - SP. In: CONGRESSO BRASILEIRO DE MEDICINA VETERINÁRIA, 26., 1999, Campo Grande. Anais...Campo Grande: 1999. p.267.

PLANO municipal de saneamento de Belo Horizonte 2004 - 2007: "saneamento para todos". Belo Horizonte: Prefeitura de Belo Horizonte, 2004. v.1, $127 \mathrm{p}$.

PROCEDIMIENTOS para estudios de prevalência por muestreo. Buenos Aires: Centro Panamericano de Zoonosis 1979. 35p. (Nota técnica ${ }^{\circ} 18$, rev.1).

REIS, R.; RYU, E.; MOPTA, J.G. et al. Pesquisa de aglutininas anti-leptospira em cães pelo teste da Microaglutinação Rápida. In: CONGRESSO BRASILEIRO DE MEDICINA VETERINÁRIA, 13., Brasília. Anais... Brasília: [s.n.] 1972. p.297.

ROCHA, B.D.; HERMANN, G.P.; MOREIRA, E.C. et al. Aglutininas anti-leptospiras em cães de uma favela do município de Belo Horizonte - MG, 2000. In: SEMANA DE INICIAÇÃO CIENTÍFICA, 11., Belo Horizonte, 2000. Anais... Belo Horizonte: UFMG, 2000.

ROSSETI, C.A.; ROMERO, G.N.; AUTERI, C.D. et al. Estudio serológico de leptospirosis em perros de partidos del Oeste del Gran Buenos Aires. Rev. Med. Vet, v.80, p.298-305, 1999.

RYU, E. Rapid microscopic agglutination test for leptospira without non-specificreaction. Bul. Off. Int. Epizoot., v.73, p.49-58, 1970.

SAMPAIO, I.B.M. Estatística aplicada à experimentação animal. Belo Horizonte: FEPMVZ, 1998. 221p.

SANTA ROSA, C.A.; TERUYA, J.M.; YANAGUITA, R.M. et al. Inquérito sorológico para leptospirose e brucelose em cães da cidade de Belo Horizonte. Arq. Esc. Vet. UFMG, v.26, p.339-342, 1974.

SANTA ROSA, C.A.; CASTRO, A.F.P.; SILVA, A.S. et al. Nove anos de leptospirose no Instituto Biológico de São Paulo. Rev. Inst. Adolfo Lutz, v.29-30, p.19-27, 1970. 